\title{
Long-Term Stability Studies for CERN-LHC
}

\author{
W. Scandale \\ CERN, CH-1211 Geneva 23, Switzerland
}

\section{Abstract}

In the modern hadron colliders, like LHC, SSC and RHIC, the stability of the single-particle motion is basically determined by the field-shape imperfections of the superconducting dipoles and quadrupoles, especially during the injection flat bottom, when the effect of the persistent currents is maximum and the transverse size of the beam is large. The non-linear fields are at the origin of two effects: the betatron tunes change with the amplitude and the momentum of the circulating particles, and, for certain combinations of the horizontal, vertical, and synchrotron tunes, non-linear resonances are excited. These phenomena have a destabilizing influence on the particle motion, over a time-scale extending up to several million turns. Some precautions can make the motion of the particles less sensitive to the non-linear components of the guiding fields. Correcting multipoles can be foreseen in the regular cells, to reduce the non-linear tuneshift caused by the systematic components of the field errors. The variations of the orbit functions can be limited along the insertions. The closed orbit and the linear coupling can be corrected sufficiently well. Finally the ripple of the power supplies can be reduced as much as possible. Most of these concepts have been embedded in the design of the LHC and their beneficial effects on the dynamic aperture have been extensively cvaluated by computer simulations.

\section{INTRODUCTION}

The motion of charged particles in circular accelerators is basically governed by the magnetic field of the guiding dipoles and the focusing quadrupoles. Intentional and non intentional non-linear fields are in general also present, the side-effect of which is to induce losses at large amplitude. Sextupoles are used to reduce the chromaticity and octupoles make the tune dependent on the amplitude, which is sometime exploited to improve the current-dependent behavior. In hadron accelerators, the destabilizing action of chromaticity sextupoles is self-compensated to a large extent due to the regularity of the lattice. However, usually, a strongly focused lattice is necessary to reduce the sensitivity to field errors, and this in turn produces two adverse effects: the strength of chromaticity sextupoles has to be increased, therefore the beam stability is reduced, and the space available for dipoles along the ring is shortened with a consequent decrease of the maximum beam energy. Unintentional multipoles due to unavoidable imperfections of the guiding and focusing fields introduce additional non-linearities, which represent the greatest hazard. However, compromises are to be found between making magnetic fields as uniform as possible and keeping the magnet cost low. This is a difficult achievement especially for superconducting magnets, whose quality depends on the mechanical tolerances of the coil geometry, rather than on the shape of the poles. Both in the Tevatron and in the Hera magnets, typical deviations from uniformity have been limited to about one part in ten thousand at 2.5 centimetres from the magnet axis. Similar values, properly extrapolated with the inner coil diameter and the superconducting filament size, are expected to be reached in the magnets of the SSC, the LHC, and RHIC.

The single-particle approach provides a sufficiently simple, reliable and coherent model of the real accelerator to investigate performances related to non-linear dynamics. The key issue is to estimate the stability of the motion over the operational cycle of the accelerator. In a linear machine with irrational tunes the motion is stable and regular all around the closed reference orbit near the magnetic axis. The non-linear fields add a tune dependence with the amplitude, which shifts tunes to rational values, provoking resonant phenomena accompanied in the phase space by islands of finite area surrounded by thin chaotic layers. The islands and the chaotic layers exist through the entire phase space. However, at small amplitude, trajectories follow invariant surfaces, the KAM tori 1 , and remain stable for indefinite time. As amplitude increases, the islands become larger until they overlap. Beyond that point, the chaotic layers become interconnected and the particle motion is no longer bounded. This is the domain in which the non-linear forces are able to provoke particle losses, that sometimes may occur after millions of turns. The border between regular and chaotic motion is called dynamic aperture. This is analytically well defined for $1 \frac{1}{2}$ dimensional $\left(1 \frac{1}{2}-D\right)$ systems only ${ }^{2}$. With more degrees of freedom, particles in stochastic layers, even close to the origin, may escape through the entire phase space, due to the so called Arnold diffusion. However, for all practical purposes, the border between mostly regular and mostly chaotic trajectories can be used as the dynamic aperture.

Both analytical and numerical tools are used to estimate the dynamic aperture as a function of various machine parameters. Improvements of the linear lattice and correction schemes are studied to reduce the influence of the non-linear forces, and to specify upper limits for the magnet imperfections. The final confirmation is in general performed with numerical simulations in which the particle position is tracked element by element around the machine for large numbers of turns.

Simpler dynamical systems, such as the Hénon map ${ }^{3}$, are advantageously used to investigate mechanisms of long-term losses.

Machine experiments with existing accelerators, in which non-linear perturbations are deliberately introduced, allow comparisons with predictions of numerical simulations. 
In the following sections three subjects are reviewed, with special emphasis for the case of the LHC: the tools by which predictions on beam stability are formulated, the particular applications on accelerator design, and finally the outcome of the experiments performed in the CERN-SPS and in the FNAL-TEVATRON.

\section{TOOLS FOR DYNAMIC APERTURE ESTIMATES}

\subsection{Tracking Simulations}

Simulations with thin-lens approximation and symplectic integrators are considered as the master tool for quantitative estimates of particle bchavior in the LHC with non-linear elements. They provide exact solutions of the equation of motion for a dynamical system approximating the accelerator. A sequence of linear transfer matrices interleaved with localized polynomial non-linearities is to be computed. Reliable results are easily obtained, since computer rounding errors can be kept under control ${ }^{4}$. However a vast computing power is required to get reliable estimates of the dynamic aperture as a function of various lattice and beam parameters. A fully realistic description of the accelerator structure is difficult if not impossible. Simplifications are also imposed by limitations in computing power. Thin-lens description of guiding and focusing fields is used, and do not imply relevant changes to orbit functions. Ignoring fringing fields, and representing non-linearities with equivalent thin-lenses is also considered acceptable.

Two computer codes are routinely used to describe LHC lattice models and compute particle trajectories of given initial conditions: MAD ${ }^{5}$, developed at CERN, and SIXTRACK 6 , developed at DESY. Both of them have scalar versions to be processed in the modern farms of workstations as well as vectorized versions to make use of modem parallel processors.

\subsection{Maps}

In linear lattices, particle coordinates can be propagated along the accelerator azimuth by Twiss transfer matrices.

The use of maps can be extended to non-linear dynamics with some precautions. This extension, originally motivated by the nced to speed-up long-term tracking simulations in hadron colliders, in fact provides a powerful tool to handle dynamical quantities, like the tune dependence with the amplitude and the momentum, the distortion functions and the smear, the high-order non-linear invariants, and finally the Fourier harmonic coefficients of the resonance driving terms. Non-lincar matrices can be constructed very efficiently with Differential Algebra techniques using Taylor expansion to some high order of algebraic operators ${ }^{7}$. One-turn Taylor maps resulting from the composition of all the linear and nonlinear elements in the accelerator, are inherently not symplectic because of the high order truncation, therefore inappropriate to preserve volume in phase space. A way to restore symplecticity, whose physical meaning, however, is not fully understood, is to replace the truncated map with a Normal Form $^{8}$, that is an integrable map, represented by a rotation of an angle depending on the amplitude of the orbit. In practice this is performed by a local change of coordinates in the phase space, which brings intricate orbits into circles. However, the normalized map has an optimized order of accuracy. Above it, the approximation is improved at lower amplitude and worsened at higher amplitude. The domain of convergency is limited by resonances of low order that are allowed by the truncated Taylor map. There are ways to handle the first limiting resonance, with Resonant Normal Forms, which are not yet made of practical use.

In the LHC, the mapping approach based on Taylor expansion and Normal Form is used to evaluate the dependence of tune-shift on the amplitude and the momentum due to systematic field-shape imperfections ${ }^{9}$. By this it is possible to identify the multipoles that are more dangerous for the stability of the motion, taking into account the quite strong high order cross terms, and to define and optimize the most suited scheme of multipolar correctors.

High order Taylor maps are also used to estimate the dynamic aperture in a faster way than with the usual element by element tracking 10 : however, in the LHC, this approach is non-controversial only for simulations up to few $10^{4}$ turns. By increasing the order of the map the violation of area preserving transport can be made arbitrarily small, but the map size grows exponentially and the computing speed decreases accordingly. An interesting result is that one can correlate the accuracy of the truncated Taylor map tracking to the size of the high order terms in the map. Alternatively, one can restore the symplecticity of the Taylor map by a linear scaling transformation to the particle coordinates at each turn. The transformation is staged in three different scale transformations, two in the transverse and one in the longitudinal directions respectively, characterized by three different scaling factors chosen in a suitable manner to ensure that the Liouville's theorem is obeyed, at least in average. In fact, there is an infinite set of possibilities for the choice of the three scaling factors. The additional criterion to determine the final choice is based on the following arguments. It is assumed that Taylor maps up to order 11 th are sufficient for an accurate description of the beam dynamics in the LHC, at least in the region of the phase-space where the motion is regular or only weakly chaotic. The beam trajectories are computed both with element-by-element tracking and with Taylor maps with initial values of the scaling factors. The difference of amplitudes in the phase-space of the two results are estimated in few iterations by slightly varying the scaling factors. From that one can optimize the scaling factors in such a way that the amplitude differences between the direct tracking and the iteration of the Taylor map is constant as a function of the number of turns. The comparison between direct tracking and iteration of truncated maps with and without dynamic rescaling has been made for a large number of turns: the agreement is two orders of magnitude better with re-scaling that without.

\subsection{Early indicators of chaos}

Early indicators of chaotic motion have been used to speed 
up the estimate of the dynamic aperture in the LHC. The exponential divergence of two initially very close trajectories is a criterion for chaos, a linear growth indicating regular motion. The exponential coefficient, called Lyapunov exponent ${ }^{11}$, can be used to localize stochastic layers in the phase space and eventually to identify the stability border below which its value is zero. The routine way to evaluate the Lyapunov coefficient is to track simultaneously two particles with a slightly different initial amplitude, and to compute periodically and plot their mutual distance ${ }^{12}$. An equivalent method is based on the analytical evaluation of the Jacobian in the phase space domain of interest ${ }^{13}$. A third possibility is to compute the slow change of an action invariant ${ }^{14}$. The predictability of all three methods is enhanced when the non-linear deformation of the phase space is removed by Lie algebraic or Normal Forms type change of coordinates. It is currently admitted that through early indicators of chaos a conservative estimate of the dynamic aperture can be obtained with about ten times less computing power than for standard tracking.

\subsection{Figure of merit and data processing}

The linear aperture, based on smear and tuneshift with the amplitude, and the short-term dynamic aperture were widely used in the past 15 to estimate non-linear effects, since threshold values for detuning and amplitude distortion were considered sufficient to ensure long-term stability. However, the validity of this extrapolation has not been confirmed by deeper studies. Therefore, intensive tracking and sophisticated data processing are preferred nowadays to estimate the dynamic aperture, after a preliminary selection of rather few significant cases, on the basis of short-term simulations. Results are presented in graphical form: survival plots depict the maximum number of stable turns as a function of starting amplitude ${ }^{16}$. These plots and early indicators of chaos provide a practical estimate of the stable region. Dense survival plots are ragged and show a large spread in the survival time close to the chaotic border, rapidly decreasing at larger amplitudes. Such an irregular shape reflects the local origin of the particle instability: at moderate amplitude in presence of weaker perturbations, the escape time is largely influenced by microscopic changes of initial coordinates; at large amplitude, instead there are only fast losses. Under the influence of nonlinearities, particles migrate across different nests of resonances, which can be at least phenomenologically correlated to average lifetime. The loss mechanism is in general sudden: the particle may stay confined even for millions of turns and then diverge in a few thousand turns.

\section{APPLICATIONS IN ACCELERATOR DESIGN}

The LHC must operate with negligible loss for long periods, up to $10^{8}$ turns, in spite of the unavoidable field shape imperfections. An upper limit to unintentional multipoles and practical compensation strategies have to be devised for a safe operation. This implies a thorough understanding of the influence of the non-linearities on the long-term behavior of particle trajectories. Analytical methods available are not yet fully exploited. Numerical simulations are too heavy and time consuming for an exhaustive overview of all the possible situations. Nevertheless, remarkable progress has been made through heuristic approaches proposed in the recent past, based on the investigation of simplified non-linear models of the LHC lattice and on the use of empiric criteria for beam stability.

Too crude simplifications of the lattice structure itself have dramatic effects on non-linear performances. Cell lattice models with only regular cells and no interaction regions show a regular azimuthal pattern of the orbit functions and in particular of the betatronic phase advance leading to unrealistic enhancement of the particle stability. They are in general used for numerical studies of simple dynamical systems as the Hénon map. A more realistic way to drop the insertions is to replace them with equivalent rotation matrices. Part of the chromatic aberration and some unintentional field errors are disregarded in this way. However, relevant informations can be gained with less computing power and complexity, especially during the injection plateau, where the stability of motion is mainly determined by non-linear perturbations in the arcs. This approach was used to determine the optimum value for the length and the phase advance of the LHC cell ${ }^{17}$, fixed to about $100 \mathrm{~m}$ and 90 degrees, respectively. More advanced studies are based on models with realistic descriptions of the insertions.

The field-shape imperfections are equivalent to multipoles up to large order, which can be expressed as the sum of two parts, one systematic and the other random. The general agreement is to stop at order 11 th in the multipolar expansion and to neglect correlations between random multipoles of different orders. Systematic errors are larger at injection due to persistent currents. Large low-order $\left(3^{\text {rd }}\right.$ and $\left.5^{\text {th }}\right)$ values provoke a sizeable detuning with the amplitude and the momentum, which can be corrected either locally 18 , as in the LHC lattice version 2, or using a clever cancellation of the detuning terms by means of Sympson rules ${ }^{19}$, as in the LHC lattice version 1. In the latter case, multipolar correctors are to be located near the main quadrupoles as well as at about the middle of each half cell ${ }^{9}$. Octupolar imperfections have, in the LHC, a particular behavior related to the symmetry of the magnetic field in two-in-one magnets: the integrated value along the azimuth is expected to be zero, therefore the detuning with the amplitude is expected to be self-compensated without specific correctors. Large high-order ( $7^{\text {th }}$ and $9^{\text {th }}$ ) systematic multipoles destabilize off-momentum particles and have to be minimized by design: tolerable values for the LHC have been found to be of the order of $2 \cdot 10^{-6}$ and $5 \cdot 10^{-7}$ units at $1 \mathrm{~cm}$ radius, respectively.

Random imperfections, which vary from magnet to magnet due to manufacturing tolerances, are the main source of nonlinear resonances and distortion functions. Statistical distributions can be easily predicted, but are insufficient for a complete knowledge of the non-linear optics, since resonance strengths depend on the specific sequence of the random errors around the ring rather than on statistical properties. Therefore, 
criteria for magnet design are to be studied on several nonlinear lattices, with different sequences of random multipoles. In fact, there are many parameters that limit the stability of the particle motion in the LHC, therefore the first task is to identify the most important ones, in order to reduce to a reasonable amount the enormous need of computing time required for an exhaustive set of simulations. Parameters routinely considered in the accelerator models are residual closed orbit, linear coupling due to imperfections, synchrotron motion, and main power supplies ripple. Chromaticity and non-linear detuning are corrected with suited set of correctors. Special cases with some residual uncompensated chromaticity are considered as well. Shortterm dynamic aperture is first evaluated by tracking particles of different starting amplitudes for $10^{3}$ turns. This is fast and well suited for a first exploration of the space parameters, and is also sufficient to reveal the most important features of the non-linear phase space. Ten different seeds are used to fix the test samples of the random errors. Appropriate subsets of them are considered to disentangle the effects of the dipole imperfections from those of the quadrupole imperfections in a machine with a perfect closed orbit and no linear coupling. By choosing three representative seeds in each distribution, i.e. one with the smallest, one with the largest, and one with an average value of the aperture, one can easily check the combined effect of the dipole and the quadrupole errors and identify a limited number of representative sets of non-linear lattice models to be investigated with long-term tracking simulations. With this strategy 20 , beam stability has been found to be strongly influenced by linear lattice parameters like tune, residual linear coupling, and peak- $B$ values in the insertion quadrupoles as well as by a residual chromaticity of a few units. Instead, residual closed orbit associated to magnet misalignment and tune ripple of a few $10^{-4}$ units showed a weak interference with beam stability.

Particles above the stable region are expected to diffuse towards the vacuum pipe at a speed strongly increasing with the transverse amplitude. A set of collimators ${ }^{21}$ is used to absorb them before they hit the magnets and provoke an unwanted deposition of energy in the superconducting coils. The transverse position of the primary collimator defines the mechanical aperture of the accelerator. It is basically fixed taking into account the mechanical tolerances of the cold bore and of the thermal screen of the main magnets, the expected peak-values of the closed-orbit, of the dispersion, and of the Bfunction modulation, and, of course, the optimum value of the separation between the primary and the secondary collimators. For a safe operation, careful matching of physical aperture and stability border is to be performed 20 . With a small mechanical aperture, trajectories with small amplitude oscillations only are allowed, which are weakly perturbed even in presence of large field-shape imperfections, whilst, with larger mechanical apertures, and larger amplitude oscillations, the non-linear perturbation becomes larger and the size of the magnetic errors start to play a leading role for the dynamic aperture. On the other hand, we believe that particles with amplitudes up to the chaotic boundary are stable, although the non-linear perturbations induce a finite smear of their trajectories, whilst particles with larger amplitudes may become unstable after a sufficiently large number of turns. Ideally, the chaotic limit should be equal to the dynamic aperture evaluated in presence of collimators, in which case only the unstable particles will be intercepted by the collimators. In this respect the mechanical size and the field quality of the LHC at injection have been found to be well matched to a value of $6 \sigma$, i.e. of $7.2 \mathrm{~mm}$, which is considered a wise choice for the needed dynamic and physical aperture.

Strategies of magnet sorting have been invented, by which the magnets are installed in such a scquence in the machines as to minimize the combined non-linear effects. For practical and theoretical reasons, the sorting scheme should be as local as possible and must refer to a limited kind of multipoles. Different solutions have been proposed 22 . By introducing a quasi-periodicity of multipoles every two betatronic wavelengths, the harmonic content of non-linearities can be shifted away from harmful frequencies. Alternatively, small groups of magnets, typically ten, are ordered in such a way to minimize a broad band of non-linear driving terms computed to 2nd perturbative order, contributing to resonances up to order 12th. The first method is used in the LHC and the SSC, the last method is used for HERA and is still under investigation for the LHC.

Diffusion with steady state increase of the amplitude has never been detected in numerical simulations, even in presence of extemal tune modulation, contrary to what is usually observed with beam-beam interaction. Due to resonance crossing and non-linear coupling, migration of particles in the tune diagram and mixing of horizontal and vertical oscillations are well visible in long-term tracking results. However large increases in amplitude and particle losses are sudden and unpredictable, even if they occur after a large number of turns.

\section{EXPERIMENTS}

In the last decade, experiments have been devised to study the effect of high-order resonances under controlled conditions. The hope is that by comparing theoretical or numerical results with experimental ones, it may be possible to define suitable criteria for beam stability and evaluate their predictive power. This is an ambitious goal, since real machines are much more complicated than models used in tracking codes or analytical evaluations. There are many phenomena, like collective instabilities, synchro-betatron resonances, linear imperfections affecting the orbit functions, the linear coupling, the closed orbit, non-linear imperfections due to fringing fields and saturations, which may take a long time to be quantified in order to disentangle single-particle effects from measurements. However, analysis of operating situations provides a wealth of informations which can be exploited to bridge the gap between models and reality. These experiments have been performed in the CERN-SPS and the FNAL-TEVATRON which are already well understood, so that clear conditions could be defined, spurious effect eliminated and phenomena under study carefully isolated, with reasonably small effort. Having repeated similar 
measurements in different accelerators is invaluable to help in distinguishing results of general interest from those which are just a property of the machine used. Common motivations of the two experiments are related to the refinement of aperture and field quality criteria for future large hadron accelerators, like LHC or SSC. A common procedure consists in exciting already existing sextupoles in order to introduce in a controlled fashion non-linearities in an otherwise linear lattice. To probe large amplitudes, a pencil beam with small emittance and momentum spread is used, to which a large enough coherent deflection is applied. In a few hundred turns, a 'hollow' distribution of charges is created around the central orbit due to nonlinear filamentation, whose behavior is observed with several instruments: current transformers record lifetime, Schottky noise detectors give tune and tune-spread, flying wires provide transverse profile, and orthogonal pairs of position monitors are able to produce a phase space portrait.

In the experiment E778 at FNAL ${ }^{23}$, sextupoles were exciting strongly the third integer resonance together with the higher-order ones. Smear, injection efficiency and short-term dynamic aperture were measured and compared with tracking. The agreement is good, however long-term losses could not be quantitatively reproduced. The existence of stable nonlinear resonance islands was demonstrated experimentally by observing coherent persistent signals of particles captured into them. Tune modulation effects were explored and compared with those of 1-D forced pendulum.

In the experiments at the SPS ${ }^{24}$, the sextupolar excitation chosen such as to minimize the third integer resonance. Detuning compensation was experimentally tested by using existing octupoles. A $30 \%$ increase of dynamic aperture resulted from a factor ten reduction of tune-spread. This provides experimental guidance in devising correction schemes for large hadron accelerators. However most of the emphasis was put on the study of slow diffusion induced by power supply ripple, and controlled modulation of a special quadrupole. The diffusion coefficient was measured as a function of the amplitude, the modulation frequency and depth, and the tune. It was obtained by scraping the beam tail with horizontal and vertical collimators, retracting them suddenly by a few $\mathrm{mm}$, and observing the beam lifetime to estimate the time taken by the particles to fill the gap created by the retraction. Diffusion immediately sets in when tune modulation is turned on, and there is evidence that a ripple which leads to tune modulation of $10^{-3}$ cannot be tolerated in a machine with strong non-linearities. A tune modulation at two frequencies is more destructive than a modulation at a single frequency for the same overall depth. The agreement with numerical simulations is of the order of $20 \%$, however the strong dependence of diffusion on modulation depth and the dependence on frequency are not yet understood.

Recent experiments at FNAL 25 have also addressed the problem of ripple induced diffusion. Intensity and transverse profile were recorded and used to deduce a phenomenological dependence of the diffusion coefficient on amplitude. The proposed model assumes a threshold amplitude, below which there is no diffusion, and above which the diffusion speed increases as a polynomial of the amplitude. A steady reduction of beam size was pointed out which was never observed at the SPS. This is likely to be typical of the regime of large losses explored at FNAL.

The phenomenological model of FNAL for diffusion has been found to be in contradiction with some results of the CERN experiment as well as with the sudden manifestation of fast amplitude growth in tracking 26 . More general models of diffusion based on Markov process with jumps, using master equations on status transition probability are under investigation.

\section{TRENDS}

Studies of theoretical aspects of the LHC related to nonlinearities are being pursued, to develop new tools for stability estimate, and to understand diffusion in 1-D and 2-D Hénon map models. However, the main tools to evaluate beam stability in the design lattice will continue to be tracking simulations and maps associated to Differential Algebra methods, whose potentiality seems to be far from being fully exploited. Experiments are likely to be vigorously pursued, to compare predictions with real world in a controlled fashion, and to clarify features of slow diffusion in presence of external modulation and non-linear fields.

\section{REFERENCES}

[1] V.I. Arnold, "Mathematical Methods of Classical Mechanics", Springer-Verlag, 1978.

[2] B.V. Chirikov, Physics Report 52 N. 5, p. 263 (1979).

[3] M. Q. Hénon, Quarterly of Appl. Math. 27, 291 (1969).

[4] E. Lohrmann, P. Wilhelm, Particle Acc. 19, p. 99 (1986).

[5] H. Grote, C. Iselin, CERN/SL/90-13 (AP) Rev.2 (1991).

[6] F. Schmidt, CERN/SL/AP 91-52 (1991).

[7] M. Berz, Particle Acc. 24, 109 (1989).

[8] A.Bazzani, et al., Il Nuovo Cimento, Vol. 102 B, N. 1 (1988).

E. Forrest, J. Irwin, M. Berz, Particle Acc. 24, p. 91 (1989).

[9] W. Scandale, et al., Particle Acc. 35, p. 53 (1991).

[10] R. Kleiss, F. Schmidt, F. Zimmermann, Particle Acc. Vol.41, N.2 (1993).

[11] A. M. Lyapunov, "Stability of Motion", Ac. Press (1966).

[12] F.Schmidt, F. Willeke, F. Zimmermann, Particle Acc. 35, p. 249 (1991).

[13] F. Zimmermann, Dipl. Thesis, DESY-HERA 91-03 (1991).

[14] J.Irwin, Y. Yan, IEEE PAC 89, p. 1340 (1989).

[15] A. Chao et al., CERN 88-04, p. 152 (1988).

[16] F. Galluccio, F. Schmidt, EPAC 90, p.640 (1990)

[17] M. Bassetti et al., IEEE PAC 87, p. 1284 (1987).

[18] M. Giovannozzi, F.Schmid, "General Normal Forms procedure to correct Tune-Shift and Non-Linear Chromaticity for Large Accelerators like the LHC", these proceedings.

[19] D. Neuffer, CERN 88-04, p. 179 (1988).

[20] Z. Guo, T. Risselada, W. Scandale, EPAC 92, p.637 (1992).

[21] Design Study of the LHC, CERN 91-03, p. 115 (1991).

[22] R. Gluckstern, S. Ohnuma, IEEE PAC 85, p. 2315 (1985). F. Willeke, DESY-HERA 87-12 (1987). W. Scandale, L. Wang, CERN SPS 89-41 (AMS) (1989).

[23] L. Merminga et al., IEEE PAC 89, p. 1429 (1989).

[24] D. Brandt et al., EPAC 90, p.1438 (1990), X. Altuna et al., CERN SL/91-41 (AP) (1991).

[25] T. Chen et al, Phys.Rev.Lett., Vol 68 N.1. p.33 (1992).

[26] A. Gerasimov, FERMILAB-Pub-92/185 (1992). 